\title{
Intrathecal enzyme replacement therapy to treat spinal cord compression in mucopolysaccharidosis: Overview and rationale
}

\author{
Agnes Chen ${ }^{\mathrm{a}, \mathrm{b}, *}$ and Patricia Dickson ${ }^{\mathrm{a}}$ \\ ${ }^{a}$ Department of Pediatrics, Harbor-UCLA Medical Center, Torrance, CA, USA \\ ${ }^{\mathrm{b}}$ Department of Neurology, Harbor-UCLA Medical Center, Torrance, CA, USA
}

Accepted for publication 21 October 2009

Keywords: Spinal cord, mucopolysaccharidosis

Spinal cord compression has been described in mucopolysaccharidosis I, II, IV, VI, and VII. The spinal cord compression in the mucopolysaccharidoses is believed to originate from narrowing of the vertebral canal due to bony abnormalities, and thickening of the meninges and spinal ligaments due to glycosaminoglycan infiltration [12]. The current standard of care involves surgical decompression. However, the surgery is extensive, MPS patients are high-risk surgical and anesthesia candidates, and the problem may recur after surgery. Neurosurgical treatment of spinal cord compression involves a multi-level decompressive laminectomy and opening of the thickened dura [12]. Many patients also require a suboccipital craniectomy. Some patients require instrumentation and fusion because they have ligamentous or spinal instability. Overall, the surgical repair can be more extensive than that required for spondylotic degeneration and myelopathy in normal elderly individuals.

*Address for correspondence: Patricia Dickson, 1124 W. Carson Street, HH1, Torrance, CA 90502, USA. Tel.: +1 310781 1399; Fax: +1 310782 2999; E-mail: pdickson@ucla.edu.
Mucopolysaccharidosis patients are risky surgical candidates due to their airway, lung, and cardiac disease. Mucopolysaccharides accumulate in the oropharynx, tongue, epiglottis, aryepiglottic folds, and tracheal wall. This can distort the anatomy and make intubation extremely difficult [21]. There have been multiple reports of emergent tracheostomies performed in the course of surgery because of airway obstruction caused by significant upper airway storage $[7,17]$. In addition, these abnormalities tend to worsen over time, making subsequent surgeries even more risky $[7,16]$. Intubation is also made more difficult by immobility of the neck, shortened neck, and cervical spine instability [21]. There is additional anesthesia risk due to obstructive and restrictive lung disease, restriction of temporomandibular joint range of motion, and cardiac disease. Intraoperative complications have been attributed to stroke, pulmonary edema, and coronary artery disease [2].

There is evidence that spinal cord compression can recur even after decompression surgery. In 1987, Tamaki et al. published a case of a 36-year-old man with Maroteaux-Lamy syndrome who underwent C1-C3 


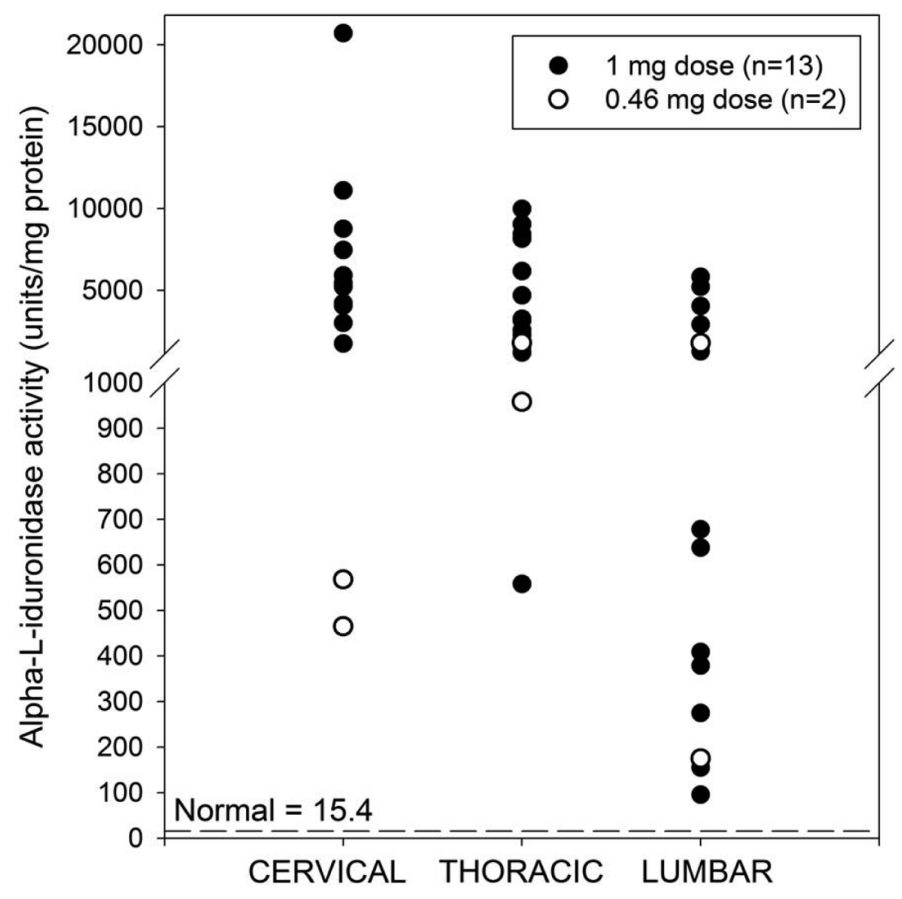

Fig. 1. Biochemical measurement of enzyme activity in cervical, thoracic, and lumbar meninges of mucopolysaccharidosis I dogs treated with 3-4 doses of intrathecal recombinant human $\alpha$-L-iduronidase weekly, monthly, or quarterly (every three months). Mean iduronidase activity in normal dogs is 15.4 units/mg protein (dashed line, $n=5$ ). These data were previously published in part $[5,13]$.

transcervical anteromedial fenestration for compression due to thickened dura [20]. He had temporary improvement, but returned with myelopathy one year later and was found to have much more severe concentric narrowing of the entire cervical region. He underwent a second surgery consisting of a C1-C7 laminectomy and duraplasty. Longer lifespan due to current therapies for systemic disease may increase the chance of recurrence in treated individuals.

Hematopoietic stem cell transplantation (HSCT) has been shown to be successful in treating many clinical symptoms of MPS I, but it is only available to the most severely affected patients given its high morbidity and mortality. In addition, many of the skeletal manifestations of the disease are probably not reversed nor prevented by HSCT. In 2000, Kachur et al. reported on an MPS I patient who received a bone marrow transplant at age two, but then presented at eight years of age with a cervical myelopathy [12]. This patient underwent a suboccipital craniectomy, $\mathrm{C} 1-\mathrm{C}-5$ laminectomy and duraplasty. She was found to have ligamentous and dural thickening, and an extradural mass comprising storage material and fibrosis.

Intrathecal or intracerebroventricular enzyme replacement therapy has been studied in animals as a potential therapy for mucopolysaccharidosis types I,
II and IIIA, globoid cell leukodystrophy (Krabbe disease), late-infantile neuronal ceroid lipofuscinosis, and Niemann-Pick type A $[4,6,8,13,14,18]$. Distribution via cerebrospinal fluid appears to be widespread in all cases. Reduction in stored material, improved histopathology, longer lifespan and neurobehavioral improvement have been observed with intrathecal or intracerebroventricular enzyme replacement therapy in animal models $[4,6,9,10,14]$.

There is a canine model of mucopolysaccharidosis type I, which has a null mutation in the IDUA gene [19]. We have published results on 19 MPS I dogs treated with intrathecal recombinant human alpha-Liduronidase (formulated as laronidase, BioMarin Pharmaceutical Inc., Novato, CA). Thirteen of these MPS I dogs received $\sim 1 \mathrm{mg}$ and six received $0.46 \mathrm{mg}$ intrathecal recombinant human alpha-L-iduronidase administered into the cisterna magna in 3-4 weekly, monthly, or quarterly (every three month) repeated injections [5, 13]. At 48 hours following the final dose, cervical, thoracic, and lumbar meninges (dura and arachnoid) were assayed for iduronidase activity and glycosaminoglycan storage levels. Iduronidase activity levels in the spinal meninges were very high, with the mean enzyme activity ranging from 33 - to 435 -fold the normal level (15.4 units/mg) in all three regions (Fig. 1). In the 


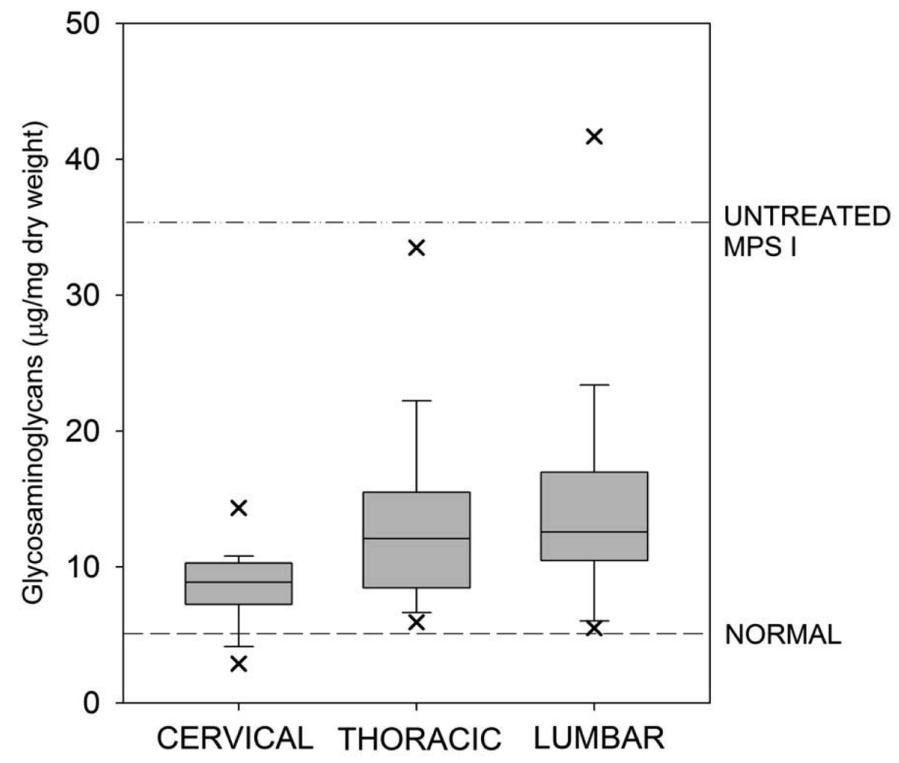

Fig. 2. Biochemical measurement of glycosaminoglycan storage in cervical, thoracic and lumbar meninges of mucopolysaccharidosis I dogs treated with 3-4 doses of intrathecal recombinant human $\alpha$-L-iduronidase weekly, monthly, or quarterly (every three months). Data shown are 25-75th percentiles (lower and upper bounds of box), median (mid-box line), 10th and 90th percentiles (error bars), and range (cross-hairs). Untreated affected dogs showed a mean glycosaminoglycan level in the meninges of $35.9 \mu \mathrm{g} / \mathrm{mg}(n=2$, upper dashed line); normal dogs showed 4.78 ( $n=4$, lower dashed line). Data were previously published in part [5,13].

cervical meninges, enzyme activity ranged from 465 to 20,700 units/mg protein (30- to over 1300 -fold normal); in thoracic meninges, 558 to 9973 units/mg (36to over 600-fold normal); in lumbar meninges, 95.4 to 5820 units/mg (6- to over 300-fold normal).

As previously reported, dogs treated with intrathecal recombinant alpha-L-iduronidase showed reduction in glycosaminoglycan storage in the cervical, thoracic, and lumbar spinal meninges by $57-70 \%$, from the untreated level of $35.9 \pm 3.03 \mu \mathrm{g} / \mathrm{mg}(n=2)$ to means of roughly $9-16 \mu \mathrm{g} / \mathrm{mg}(n=19)$ [5]. Normal total glycosaminoglycan content in the spinal meninges was $4.78 \pm 0.82(n=4)$. There was no difference between the $1 \mathrm{mg}$ and $0.46 \mathrm{mg}$ dose in glycosaminoglycan storage reduction. Mean glycosaminoglycan storage in the cervical meninges was reduced to a greater extent than thoracic or lumbar, and overlapped with the normal range (Fig. 2). Six of the 19 dogs were evaluated three months after the final intrathecal laronidase dose. Glycosaminoglycan storage was equally low in these animals as at 48 hours [5]. Histopathology showed storage reduction in the meninges.

An immune response occurs to intrathecal enzyme administration in animals. Mice with mucopolysaccharidosis IIIA treated with intrathecal sulfamidase showed no cellular immune response (meningitis), but all mice developed antibodies in serum [10]. Mice treated with intraventricular tripeptidyl peptidase-1 for late-infantile neuronal ceroid lipofuscinosis had no apparent side effects, but also developed antibodies to therapy [4]. In our dogs with mucopolysaccharidosis type I, intrathecal enzyme therapy resulted in a lymphocytic and plasmocytic infiltrate in the meninges, accompanied by anti-iduronidase antibodies in serum and cerebrospinal fluid $[5,13]$. There was no clinical evidence of meningitis in the animals, however. Induction of immune tolerance, or even partial immune suppression, may reduce the cellular and humoral response, though less frequent intrathecal injections may also be a factor [5].

Preliminary clinical trials to determine the safety of this approach in patients with mucopolysaccharidosis type I are underway (NCT 00215527 and NCT 00786968). The goal is to study patients with symptomatic spinal cord compression who are not in urgent need of surgical intervention and whose compression is not mainly due to extradural factors (such as spinal disk intrusion). The initial pilot study is four months' duration, with a one-year extension for subjects with measureable gains from therapy. Subjects receive intrathecal laronidase injections of $1.74 \mathrm{mg}$ diluted in Elliotts B artificial spinal fluid (total volume $9 \mathrm{~mL}$ ) via lumbar spinal tap. Evaluations include serial neurologic examinations, six-minute walk test, modified Japanese or- 
thopedic association scoring for myelopathy, functional independence measure, magnetic resonance imaging of the cervical spine, and somatosensory evoked potentials of the upper and lower extremity $[1,3,11,15$, 22].

The clinical effects of intrathecal enzyme replacement therapy for spinal cord compression in mucopolysaccharidosis may be difficult to assess. Thickening of extradural ligaments is a major contributor to cervical cord compression; this is not expected to respond to intrathecal enzyme replacement therapy, and cannot reliably be differentiated from dural thickening on spinal imaging. Other extradural factors also contribute to cord compression, and can be the primary cause. Damage to the spinal cord is typically irreversible, making the measurement of success difficult. Long-standing glycosaminoglycan storage can lead to tissue damage and fibrosis, which may not respond to therapy. Prevention of damage may be a more achievable goal, but this is very hard to assess clinically, as it would require very long-term, controlled studies.

\section{Acknowledgements}

This manuscript was developed as the result of a meeting of experts entitled "Promoting Bone Health in MPS VI: Framing New Therapies" held in Oakland, California in October, 2008. This meeting was supported by an educational grant from BioMarin Pharmaceutical, Inc., Novato, CA. BioMarin had no role in the content presented and discussed at the meeting. All authors participated in the development and writing of the manuscript and are fully responsible for its content. The research described is funded by grants from BioMarin, Genzyme Corp., the Ryan Foundation for MPS Children, the Food and Drug Administra tion (FD003450) and the National Institutes of Health (NS054242).

\section{Conflicts of interest}

The authors receive research support from Biomarin Pharmaceutical and Genzyme Corp., maker and distributor (respectively) of laronidase. Patricia Dickson also receives research support from Shire Human Genetic Therapies and Zacharon Pharmaceuticals. Biomarin, Genzyme and Shire have paid for travel to scientific meetings for Patricia Dickson. The Los Angeles Biomedical Research Institute at Harbor-UCLA Medical Center and the Department of Pediatrics (but not the authors) receive royalties from the sale of laronidase.

\section{References}

[1] ATS Statement: Guidelines for the Six-Minute Walk Test, $A m$ J Respir Crit Care Med 166(1) (2002), 111-117.

[2] K.G. Belani, W. Krivit, B.L. Carpenter, E. Braunlin, J.J. Buckley, J.C. Liao et al., Children with mucopolysaccharidosis: Perioperative care, morbidity, mortality, and new findings, $J$ Pediatr Surg 28 (1993), 403-408.

[3] R. Boor, E. Miebach, K. Brühl and M. Beck, Abnormal Somatosensory Evoked Potentials Indicate Compressive Cervical Myelopathy in Mucopolysaccharidoses, Neuropediatrics 31(3) (2000), 122-127.

[4] M. Chang, J.D. Cooper, D.E. Sleat, S.H. Cheng, J.C. Dodge, M.A. Passini et al., Intraventricular Enzyme Replacement Improves Disease Phenotypes in a Mouse Model of Late Infantile Neuronal Ceroid Lipofuscinosis, Mol Ther 16(4) (2008), 649-656.

[5] P. Dickson, M. McEntee, C. Vogler, S. Le, B. Levy, M. Peinovich et al., Intrathecal enzyme replacement therapy: successful treatment of brain disease via the cerebrospinal fluid, Mol Genet Metab 91(1) (2007), 61-68.

[6] J.C. Dodge, J. Clarke, C.M. Treleaven, T.V. Taksir, D.A. Griffiths, W. Yang et al., Intracerebroventricular infusion of acid sphingomyelinase corrects CNS manifestations in a mouse model of Niemann-Pick A disease, Experimental Neurology 215(2) (2009), 349-357.

[7] L. Gaitini, M. Fradis, S. Vaida, G. Collins, M. Croitoru, M. Somri et al., Failure to control the airway in a patient with Hunter's syndrome, J Laryngol Otol 112 (1998), 380-382.

[8] A.R. Garcia, J.M. DaCosta, J. Pan, J. Muenzer and J.C. Lamsa, Preclinical dose ranging studies for enzyme replacement therapy with idursulfase in a knock-out mouse model of MPS II, Molecular Genetics and Metabolism 91(2) (2007), 183-190.

[9] K.M. Hemsley, H. Beard, B.M. King and J.J. Hopwood, Effect of high dose, repeated intra-cerebrospinal fluid injection of sulphamidase on neuropathology in mucopolysaccharidosis type IIIA mice, Genes, Brain and Behavior epub ahead of print, 2008.

[10] K.M. Hemsley, B. King and J.J. Hopwood, Injection of recombinant human sulfamidase into the CSF via the cerebellomedullary cistern in MPS IIIA mice, Molecular Genetics and Metabolism 90(3) (2007), 313-328.

[11] J.K. Houten and P.R. Cooper, Laminectomy and Posterior Cervical Plating for Multilevel Cervical Spondylotic Myelopathy and Ossification of the Posterior Longitudinal Ligament: Effects on Cervical Alignment, Spinal Cord Compression, and Neurological Outcome, Neurosurgery 52(5) (2003).

[12] E. Kachur and R. Del Maestro, Mucopolysaccharidoses and spinal cord compression: case report and review of the literature with implications of bone marrow transplantation, $\mathrm{Neu}$ rosurgery 47 (2000), 223-228.

[13] E. Kakkis, M. McEntee, C. Vogler, S. Le, B. Levy, P. Belichenko et al., Intrathecal enzyme replacement therapy reduces lysosomal storage in the brain and meninges of the canine model of MPS I, Mol Genet Metab 83(1-2) (2004), $163-174$.

[14] W.C. Lee, Y.K. Tsoi, F.J. Troendle, M.W. DeLucia, Z. Ahmed, C.A. Dicky et al., Single-dose intracerebroventricular administration of galactocerebrosidase improves survival in a mouse model of globoid cell leukodystrophy, FASEB J 21(10) (2007), 2520-2527.

[15] F.J. Maynard, M. Bracken, G. Creasey, J.J. Ditunno, W. Donovan, T. Ducker et al., International Standards for Neurological 
and Functional Classification of Spinal Cord Injury. American Spinal Injury Association, Spinal Cord 35(5) (1997), 266-274.

[16] C. Moores, J.G. Rogers, I.M. McKenzie and T.C. Brown, Anaesthesia for children with mucopolysaccharidoses, Anaesth Intensive Care 24 (1996), 459-463.

[17] M.J. Ruckenstein, R.E. Macdonald, J.T. Clarke and V. Forte, The management of otolaryngological problems in the mucopolysaccharidoses: a retrospective review, J Otolaryngol 20 (1991), 177-183.

[18] P.S. Savas, K.M. Hemsley and J.J. Hopwood, Intracerebral injection of sulfamidase delays neuropathology in murine MPSIIIA, Molecular Genetics and Metabolism 82(4) (2004), 273285.

[19] L.J. Stoltzfus, B. Sosa-Pineda, S.M. Moskowitz, K.P. Menon, B. Dlott, L. Hooper et al., Cloning and characterization of cD-
NA encoding canine alpha-L-iduronidase. mRNA deficiency in mucopolysaccharidosis I dog, J Biol Chem 267(10) (1992), 6570-6575.

[20] N. Tamaki, N. Kojima, M. Tanimoto, T. Suyama and S. Matsumoto, Myelopathy due to diffuse thickening of the cervical dura mater in Maroteaux-Lamy syndrome: report of a case, Neurosurgery 21 (1987), 416-419.

[21] A.H. Yeung, M.J. Cowan, B. Horn and K.W. Rosbe, Airway management in children with mucopolysaccharidoses, Arch Otolaryngol Head Neck Surg 135 (2009), 73-79.

[22] K. Yonenobu, K. Abumi, K. Nagata, E. Taketomi and K. Ueyama, Interobserver and Intraobserver Reliability of the Japanese Orthopaedic Association Scoring System for Evaluation of Cervical Compression Myelopathy, Spine 26(17) (2001). 